\title{
Genomic Analysis and Comparison of Pakistani Camels (Camelus dromedarius) by Prion Gene
}

\author{
Tanveer Hussain ${ }^{1}$, Muneeb Musthafa ${ }^{2}$, Masroor Ellahi Babar', Faiz Marikar ${ }^{3}$, \\ Fiaz Hussain', Saeed Akram Khan', Shahid Sherzada' \& Ahmad Ali ${ }^{4}$
}

\begin{abstract}
Background: In many parts of the Old World, domesticated camels (genus - Camelus) are an essential resource, providing food, labor, commodities, and sport to millions of people Of the three extent species, two have been domesticated (singlehumped dromedarius, Camelus dromedarius, and two humped Bactrian camels Camelus bactrianus) and one remains wild (two-humped wild Bactrian camels Camelus ferus). All three species possess a variety adaptations to harsh desert conditions, including mechanisms to tolerate of extreme temperatures, dehydration, and sandy terrain. People residing in harsh climate zones of the world are being benefitted by raising camels in terms of draft, milk, meat, hides and wool from centuries. There are different breeds of dromedary camels distributed in various parts of Pakistan; however there have been scarcity of research work on camels in Pakistan. Identification of novel link between Camel breeders with fatal neurodegenerative disorders is presence or not can be detect by a Prion gene and it was not carried out in Pakistan soil to date. Prion diseases which are a group of fatal neurodegenerative disorders affect both animals and humans. It is believed that the prions are infectious agents responsible for transmissible spongiform encephalopathies. In this study we report the first study on Prion protein gene in dromedary camels of Pakistan.

Material, Methods \& Results: Genes are the blueprint of life and determine the functional aspects of cellular mechanisms. Genomic DNA of the enrolled blood samples was extracted using the Nucleospin ${ }^{\circledR}$ DNA extraction kit. Genomic DNA was run on Agarose gel electrophoresis, checked the Genomic DNA quality and amplified using prion region specific primer pair. Prion protein gene was amplified $(770 \mathrm{bp}$ ) in 35 individuals of seven dromedary camel breeds from the province Balochistan and Punjab of Pakistan. Samples having required fragment size were selected and sent for sequencing through Sanger Sequencing. All the sequences were aligned through multiple sequence alignment and edited using Codon Code aligner and explored for phylogenetic analysis. A portion of $667 \mathrm{bp}$ was finally selected for phylogenetic analysis of dromedary camels from Pakistan with 61 different mammalian species (drawn from GenBank) that revealed five different clades. We found $99.9 \%$ nucleotide sequence similarities among Dromedary camels (Germany), Dromedary camels (Iran), and Dromedary camels (Pakistan). We observed deletion in dromedary camels in codon region 66-69 except wild Bactrian camels that might be the causative factor for Prion protein gene resistance in camels. The Neighbor-Joining method with bootstrap analysis of 1000 replicates was used to draw phylogenetic tree.

Discussion: This study documents the presence of 14 PrP polymorphisms and shows the relationship between different camel breeds. The deletion had not previously been examined PrP allelic variation, and was found to segregate in these breeds. However, additional data are necessary to demonstrate PrP and genetic approach will be ideal for the future studies and, more investigations are necessary to demonstrate PrP genetic resistance in camels. The utility of these techniques in identifying Prion genes and selecting superior animals and culling the weak animals and making them parents of the next generation that will lead to producing more meat and milk with fewer animals are well discussed and by slaughtering of high Prion incidence will eliminate human animal fatal neurodegenerative disorders.
\end{abstract}

Keywords: dromedary camels, prion protein gene, polymorphism, phylogenetic, Pakistan. 


\section{INTRODUCTION}

Camels have been grouped to class Mammalia, order Artiodactyla and family Camelidae [1,14]. Camelus genus contains two species domesticated, C. bactrianus, and $C$. dromedarius, one remains wild C. ferus [7,10,29]. The new world camel's classification is still a matter of debate where genus Vicugna and genus Lama [6,9,28,29]. The camels are able to survive in life-threatening drought periods, so that their reproduction and production can linger on $[7,8,26]$. Furthermore, camels have a significant role in transportation in rural civilizations [7,8,14,26,28].

Prions are infectious agent different from the rest of others due to their mode of replication, nature and disease $[12,20]$. Genes encoding prion proteins seem to be existed in higher species of animals, including mammals and avian $[12,13,17,18,21,30]$. Prions proteins cause serious neurodegenerative disorder in animals and humans due to transformation of cellular prion protein $\left(\mathrm{PrP}^{\mathrm{c}}\right)$ into pathological isoform $\left(\mathrm{PrP}^{\mathrm{sc}}\right)$ central nervous system causes neurodegenerative disease $[11,12,22,23,27]$.

Prion $\operatorname{PrP}$ is similar to the susceptibility and regulate species barrier in prion disorder [15,25]. Prions in mammals are linked to the onset of transmissible spongiform encephalopathy [2]. Upon commencement of transmission, conformational changes occur in animal prion protein $[16,24]$. The incident of transmission of disease mainly depends on genotype, mammalian breed, exposure and succestibility to the infectious agent [3]. The main objective of this study is to identify the genetic analysis and relationship of Pakistani Camels (Camelus dromedarius) interrogated by PrP Gene.

\section{MATERIALS AND METHODS}

\section{Animal selection}

Two humped camels are small in numbers and typically found in northwest part of the country. One humped Camelius dromedarius species of Pakistani camels was selected for the current study. All the enrolled subjects were healthy and belonged to different geographical regions of Pakistan.

\section{Blood Sample Collection}

Blood samples of all the enrolled subjects were collected from different geographical regions of Pakistan. The Venous blood samples were collected in $50 \mathrm{~mL}$ falcon tube having anticoagulant (EDTA) and brought to the laboratory for further analysis. The blood samples were stored in $-20^{\circ} \mathrm{C}$ maintained freezer until required.
Genomic DNA Isolation and PCR

Genomic DNA of the enrolled blood samples was extracted using the Nucleospin ${ }^{\circledR}$ DNA extraction kit ${ }^{1}$ according to the manufacturer protocol. Genomic DNA was amplified using prion region specific primer pair. The Sequences of prion region specific primers used were as follow: forward primer (5'ATCCTGGTTCTCTTTGTGGT3') and reveres primer (5'CCCACTATGAGGAAA ATGAG3'). PCR analysis was carried out by using $25 \mu \mathrm{L}$ of erection mixture containing $2 \mu \mathrm{L}$ of template DNA, 2 $\mu \mathrm{L}$ of DNTPs (20 mM each), $2 \mu \mathrm{L}$ 10X PCR buffer, $2 \mu \mathrm{L}$ $50 \mathrm{mM} \mathrm{MgCl} 2,1 \mu \mathrm{L}$ of each forward and reverse primer (10 pM), 0.5 $\mu \mathrm{L}$ Taq Polymerase ${ }^{2}$ and $14.5 \mu \mathrm{L}$ ddH2O. Thermocycler conditions used for amplification were: initial denaturation at $94^{\circ} \mathrm{C}$ for $3 \mathrm{~min} ; 35$ cycles of denaturation at $94^{\circ} \mathrm{C}$ for $45 \mathrm{~s}$, annealing at $54^{\circ} \mathrm{C}$ for $45 \mathrm{~s}$, extension at $72^{\circ} \mathrm{C}$ for $45 \mathrm{~s}$ followed by final extension at $72^{\circ} \mathrm{C}$ for $10 \mathrm{~min}$. Amplified PCR products were electrophoresed in $1.2 \%$ agarose gel, stained with Ethidium Bromide and visualized under Gel documentation system ${ }^{3}$.

\section{Sequencing and Phylogenetic Analysis}

Samples having required fragment size were selected and sent for sequencing through $1^{\text {st }}$ Base, Singapore. All the sequences were aligned and edited using Codon Code aligner and finally 638 bp were explored for phylogenetic analysis. Basic Local Alignment Search Tool was used to confirm the identity of the sequences. The PrP sequences of various animals were obtained from NCBI (National Center for Biotechnology Information US). The obtained sequences were analyzed first to exclude frame shift or vague portion. The PrP published sequences of 61 animals from different parts of the world were used to compare and analyze with the one humped dromedary camels of Pakistan. MEGA version 6 was used for molecular evolution and phylogenetic analysis. The Neighbor-Joining method with bootstrap analysis of 1000 replicates was used to draw phylogenetic tree.

\section{RESULTS}

Fourteen polymorphisms were observed in six types of camels PrP, named as Bacterian camel (China), Dromedary camel (Germany), Dromedary camel (Iran), Dromedary camel (Pakistan), Dromedary camel (UK) and Wild Bacterian camel. The amino acid substitution in codons 49, 50, 53, 60, 65, 66, 67, 68, 69, 70, 71, 103, 104 and 219 and their configurations are shown in Figure 1.Dotted areas are conserved regions of the gene and highlighted with the relevant polymorphisms. 
T. Hussain, M. Musthafa, M. Babar, et al. 2017. Genomic Analysis and Comparison of Pakistani Camels (Camelus dromedarius) by Prion Gene.

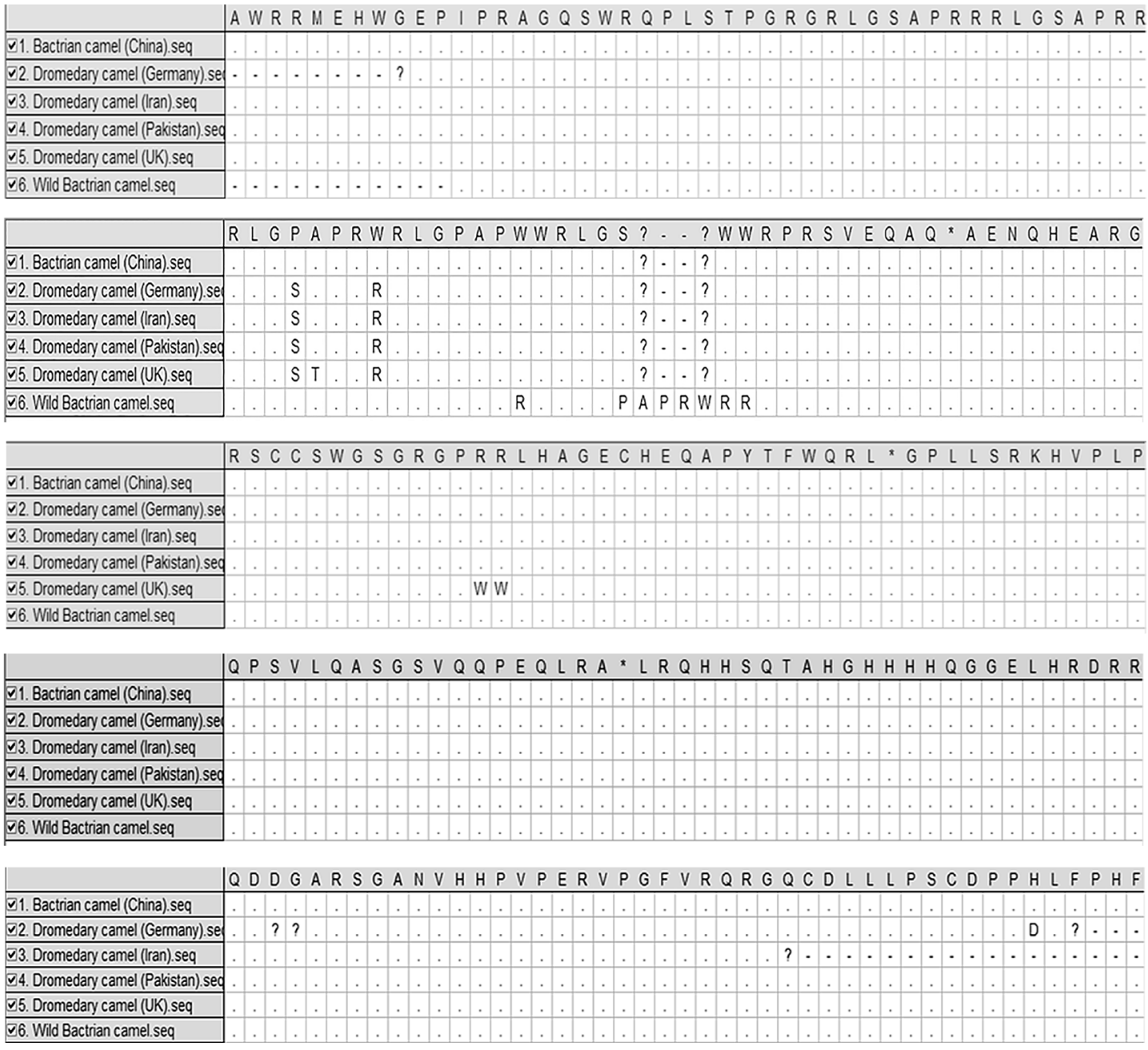

Figure 1. Alignment of the amino acid sequences of the prion protein gene of six types of camels including Pakistan. Sequence dissimilarity is indicated and the conserved regions are denoted by a dot.

Therefore, genetic analysis of the PrP nucleotide sequence from camels will trace the evolution process and especially camel breeds of Pakistan might have a high potential when compared with other breeds from all over the world. In the present study, PrP nucleotide sequence of 61 different mammalian species from all over the world were aligned with single humped camels of Pakistan and phylogenetic tree was constructed using MEGA6 software comprises of 5 Clads, A, B, C, D and E starting from the base of tree, which are further divided into different subclads and each subclad also has different nodes and animals at their tips (Figure 2). Phylogenic tree presented in Figure 2 suggested that, among all 61 species, which were given in the tree the highest similarity was observed with the hippopotamus and the lowest similarity with harbour seal. Amino acids percentages of six camels were given in Table 1, and it reflects that Iran and Pakistan camels' amino acids percentage varies dramatically. Further each node has a good value of support.
The clad A is further sub divided into 5 subclads: A1, A2, A3, A4 and A5. The subclad A1 comprises of 9 animals, Skunk bear at the bottom and the American black bear at the top, which shows that Skunk bear is the oldest breed of this subclad. Also this skunk bear is at the bottom of the phylogenetic tree, representing the ancestor of all animals. The second subclad A2 has three animals: harbour seal at the bottom and walnus at the top, denoting that harbour seal is the oldest of these three animals. Subclad A3 is consists of small Asian Mongoos which are equally related to all animals of subclad A4 having 6 animals in this clad: cloudal leopard at the bottom and Canada lynx at the top. The subclad A5 has two very closely related animals: Bit fox and Grey wolf. The clad B is divided into two subclads B1 and B2. B1 has 6 animals: wild horse is the oldest and Grans Zebra is the successor. Animals in B2 is equally related with B1 animals. Clad C has three subclads C1, C2 and C3. C1 has Pig only while 5 animals are included in C2 subclad: Dromedary camel 
of Pakistan at the bottom and Bactrian camel of china at the top the subclad C3 has 3 animals in which Alpaca is equally related to two very close animals (Llama and wild Bactrian camel). The three animals of $\mathrm{C} 2$ sub clad Dromedary camel of Pakistan, Iran and Germany are identical in genotypes. The clad D again comprises of two subclads D1 and D2, D1 has and the single animal Hippotamous while D2 has 6 animals: bottlenose dolphin, the ancestor and Minke whale, the successor of this subclad. The last Clad $\mathrm{E}$ is also divided into four subclads: E1, E2, E3 and E4 and each subclad comprises of 3, 7, 4 and 8

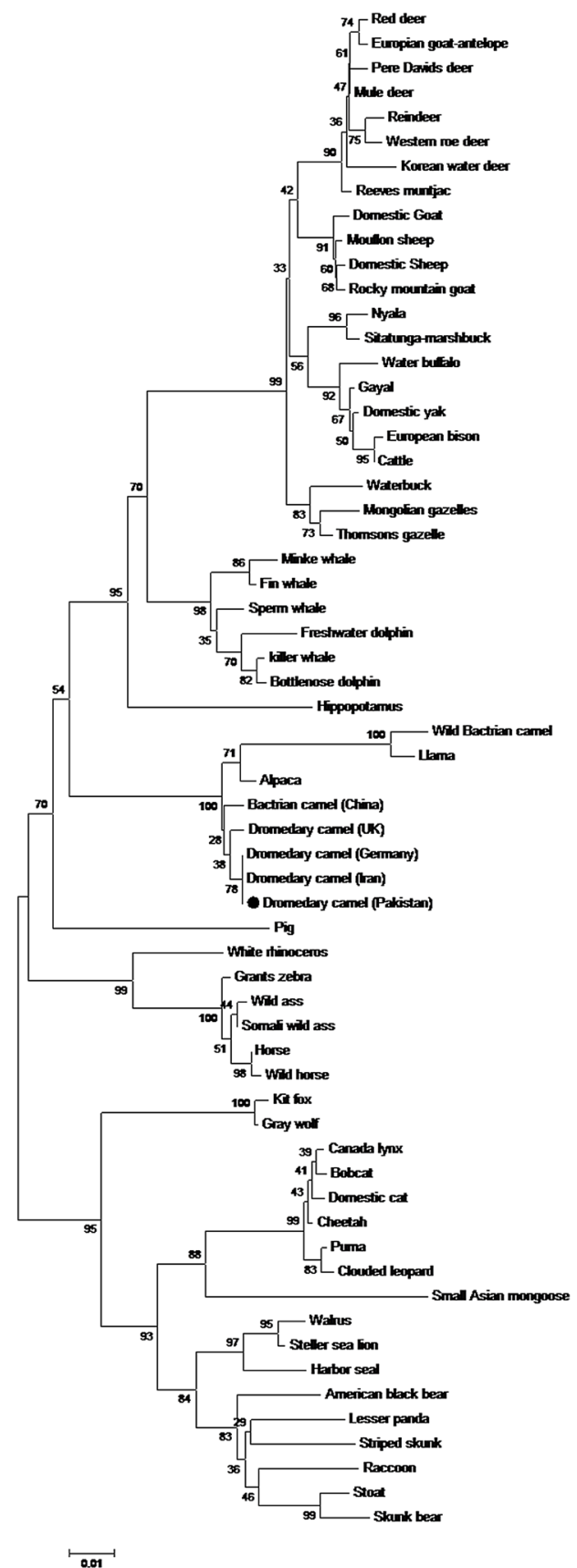

Figure 2. Molecular Phylogenetic analysis by Maximum Likelihood Method of animals of different origin animals respectively. Thomson Gazelle is the ancestor while Red deer is the latest animal of the clad E, and it is also the successor of all animals included in this phylogenetic tree and Skunk bear is the ancestor of all animals presented.

After alignment we found that at certain positions for example at 102 there is deletion of an amino acid glycine in all camels. At 109 there is amino acid alanine in camels while there is serine or threonine in other species. At position 111 there is Glycine in all camels and some other species but serine is also present in some species. At position 122 all camels have serine while other species have asparagines. At position 245 all camels have tyrosine while all the other species have serine at that position. At position 250 all camels have serine while other species have tyrosine at that position. At position 252 all camels have glycie amino acid while other species have glutamine at that position. These are the main polymorphisms which can be seen between camels and other species and should be responsible for resistant against prion diseases in camels (Data not shown).

Further, for the confirmation of the relationship among the camels, we separated the tree only with 6 types of camels and drew the phylogenetic tree. According to Figure 3, it is evident that Dromedary camel (Germany), Dromedary camel (Iran) and Dromedary camel are (Pakistan) in one Clads, Dromedary camel (UK) in another Clad and finally wild Bacterian camel and Bacterian camel (China) are in a different Clads.

The number of amino acid substitutions per site from between sequences is shown in the Table 2 . Analyses were conducted using the Poisson correction model which estimated of evolutionary Divergence between sequences [1]. The analysis involved 6 amino acid sequences. All positions containing gaps and missing data were eliminated for this study. There were a total of 187 positions in the final dataset. Evolutionary analyses were conducted in MEGA5 [2]. It further confirms that our hypothesis is correct, which Dromedary camel (Germany), Dromedary camel (Iran), and Dromedary camel (Pakistan) evolved in somewhere Arab region or Pakistan. According to the phylogenic tree the Figure 3 and Table 2 confirms that wild Bacterian camel conserved region is the highest percentage and it will be the oldest camel of evolution and the rest might have evolved by breeding. 

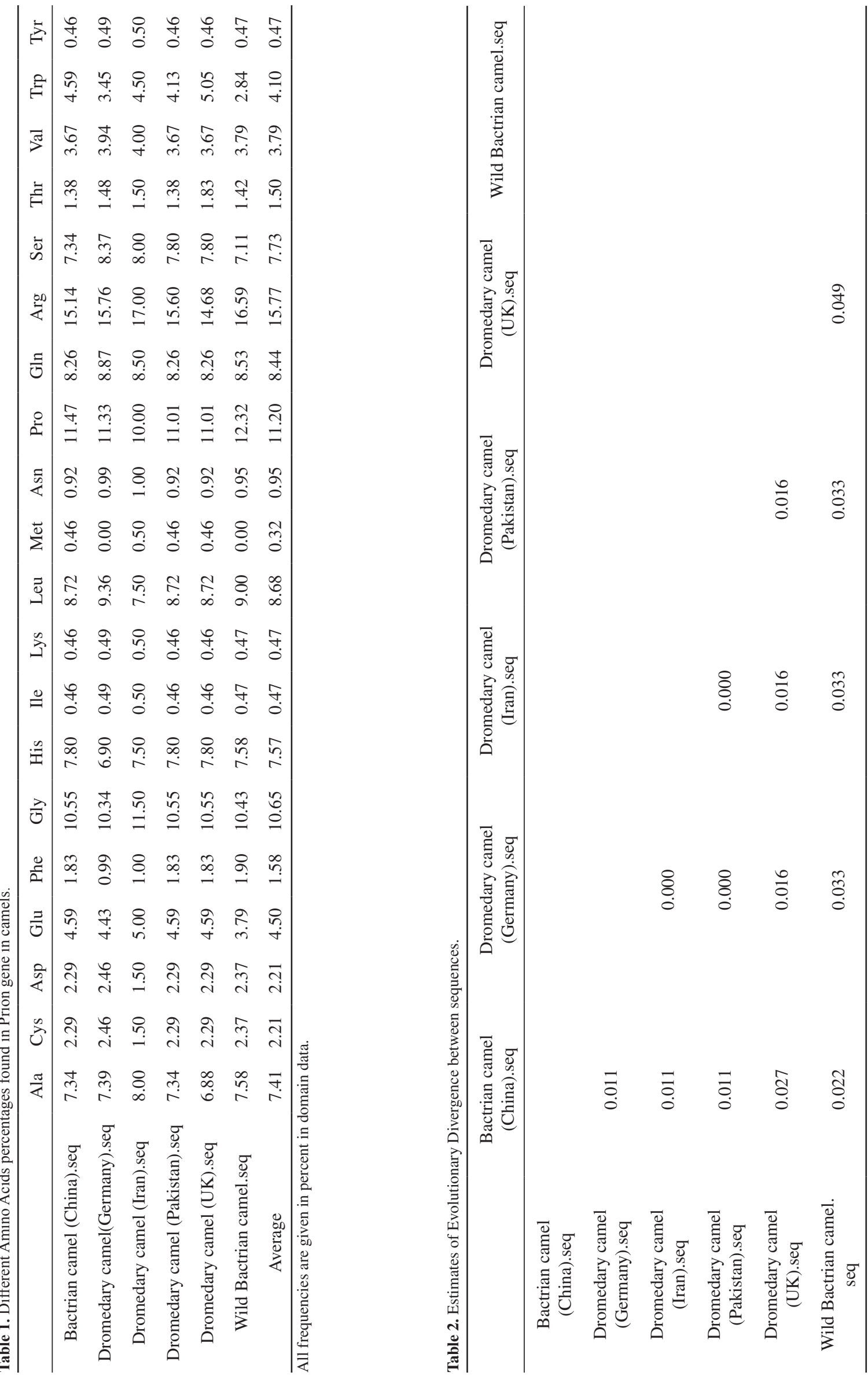


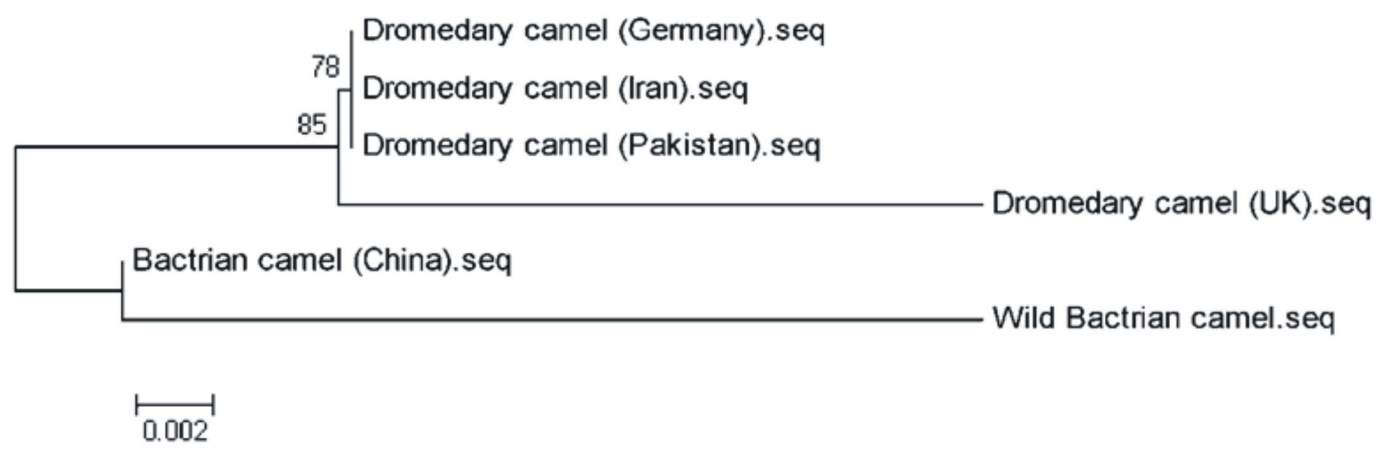

Figure 3. Molecular Phylogenetic analysis by Maximum Likelihood Method of Camel breeds.

\section{DISCUSSION}

The prion protein is coded by PRNP gene, which is active in brain and several other tissues. The proposed roles of prion protein are the transport of copper into cells and protection of brain cells (neurite outgrowth and neuronal survival) [3] also formation of synapses (synaptic function) [4]. The prion protein cellular ( $\mathrm{PrPc}$ ) during conversion into PrPSc undergoes substantial structural rearrangement by post translational modification mechanism). However, molecular structure of prion protein scrapie is poorly understood [5,19]. During the present study the published prion protein sequences (amino acid sequences) were obtained from NCBI five different species and Pakistan was sequenced in which prion disease, which was more common in camels. Furthermore, till to date Prion has never been reported in camel, due to some reasons such as PrP sequence in camel as compared to mouflon (Ovis aries) and Cattle (Bos taurus) in which there are more reports of scrape and Mad Cow Disease. These sequences were aligned using CLUSTAL W software with the different camel breeds to find out the variability between these.

As the first step of this study, this was revealed that 14 different PrP polymorphisms were present. In this study it was revealed that all six camels codon region 66-69 deletion was observed with the evolution, and it was conserved only in Wild Bacterian camel. This might be a good indicator for determination of the nucleotide sequence of the PrP camels from all parts of the world. To find out the degree of diversity in the genetics in PrP gene, we performed the multiple sequence alignment (Figure 1); 99.9\% similarities were found in nucleotide sequence comparison based upon alignment among Dromedary camel (Germany), Dromedary camel (Iran), and Dromedary camel (Pakistan). It is not clear from where Dromedary camel (Germany) migrated with the record of history. A small difference was observed six camels evolutionary in this samples which highlight relationship suggesting a simple evolutionary history (Table 2 \& Figure 3).

Multiple sequence alignment reflects the main polymorphisms that can be seen between camels and other species, and should be responsible for resistant against prion diseases in camels. Species barrier was associated with the degree of sequence homology, homology in amino acid sequence and its three dimensional structure of PrP among different species as demonstrated by previous studies on transgemic mice $[3,30]$. TSE outbreak is neither reported both in two humped and in one humped camel. In camels there is limited information available regarding prion gene variability [4]. The expression of prion protein in host e.g sheep, deer, and human is affected largely by polymorphism in PrP gene. The susceptibility and phenotypic expression of the disease are both attributed to polymorphism in codon 129 which results in either valine or methionine [19].

\section{CONCLUSION}

In summary, this study documents the presence of 14 PrP polymorphisms and shows the relationship between different camel breeds. The deletion had not previously been examined PrP allelic variation, and was found to segregate in these breeds. However, additional data are necessary to demonstrate PrP and genetic approach will be ideal for the future studies.

\section{MANUFACTURERS}

${ }^{1}$ Macherey-Nagel GmbH \& Co. KG. Düren, Germany.

${ }^{2}$ Thermo Scientific. Waltham, MA, USA.

${ }^{3}$ Bio Red. Richmond, CA, USA. 
Ethical approval. According to the Guidelines for the Care and Use of Animals of Virtual University of Pakistan, this study was carried out, and animal experiments were approved by Care and Use of Animals Center, Virtual University of Pakistan.
Declaration of interest. The authors declare that they have no conflict of interest. The authors alone are responsible for the content and writing of paper.

\section{REFERENCES}

1 Arnason U. \& Janke A. 2002. Mitogenomic analyses of eutherian relationships. Cytogenetic and Genome Research. 96: 20-32.

2 Borchelt D.R., Scott M., Taraboulos A., Stahl N. \& Prusiner S.B. 1990. Scrapie and cellular prion proteins differ in their kinetics of synthesisand topology in cultured cells. The Journal of Cell Biology. 110: 743-752.

3 Chen S.A, Mange A., Dong L., Lehmann S. \& Schachner M. 2003. Prion protein as trans-interacting partner for neurons is involved in neurite outgrowth and neuronal survival. Molecular and Cellular Neuroscience. 22: 227-233.

4 Collinge J., Whittington M.A., Sidle K.C., Smith C.J., Palmer M.S., Clarke A.R. \& Jefferys J.G. 1994. Prion protein is necessary for normal synaptic function. Nature. 370: 295-297.

5 Diaz-Espinoza R. \& Soto C. 2012. High-resolution structure of infectious prion protein: the final frontier. Nature Structural \& Molecular Biology. 19: 370-377.

6 Kadwell M., Fernandez M. \& Stanley H.F. 2001. Genetic analysis reveals the wild ancestors of the llama and the alpaca. Proceedings of the Royal Society Biological Sciences. 268(1485): 2575-2584.

7 Mukasa-Mugerwa E. 1981. The Camel (Camelus dromedarius): A Bibliographical Review. Monograph 5. Addis Ababa: ILCA (International Livestock Centre for Africa), 147p.

8 Nagarajan G.S., Shelesh K., Ghorui S.K., Pathak K.M.L., Singh R.K. \& Patil N.V. 2012. Cloning and sequence analysis of IL-2, IL-4 and IFN- $\gamma$ from Indian Dromedary camels (Camelus dromedarius). Research in Veterinary Science. 92: 420-426.

9 Penedo M.C.T., Fowler M.E., Bowling A.T., Anderson D.L. \& Gordon L. 1988. Genetic variation in the blood of llamas, Llama glama, and alpacas, Llama pacos. Animal Genetics. 19: 267-276.

10 Potts D.T. 2004. Camel Hybridization and the Role of Camelus bactrianus in the Ancient Near East. Journal of the Economic and Social History of the Orient. 47: 143-165.

11 Prusiner S.B. 1998. Prions. Proceedings of the National Academy of Sciences of the United States of America (PNAS). 95: 13363-13383.

12 Prusiner S.B. 1991. Molecular biology of prion diseases. Science. 252: 1515-1522.

13 Rivera-Milla E., Stuermer C.A. \& Malaga-Trillo E. 1992. An evolutionary basis for scrapie disease: identification of a fish prion mRNA. Trends in Genetics. 19: 72-75.

14 Schwartz H.J. \& Dioli M. 1982. The one-humped camel in Eastern-Africa. Cape Town: Verlag, pp.25-55.

15 Scott M., Foster D., Mirenda C., Serban D., Coufal F., Wälchli M., Torchia M., Groth D., Carlson G. \& DeArmond S. 1989. Transgenic mice expressing hamster prion protein produce species-species-scrapie infectivity and amyloid plaques. Cell. 59: 847-857.

16 Silveira J.R. 2005. The most infectious prion protein particles. Nature. 437: 257-261.

17 Simonic T., Duga S., Strumbo B., Asselta R., Ceciliani F. \& Ronchi S. 2000. cDNA cloning of turtle prion protein. FEBS Letter. 469: 33-38.

18 Strumbo B., Ronchi S., Bolis L.C. \& Simonic T. 2001. Molecular cloning of the cDNA coding for Xenopuslaevis prion protein. FEBS Letter. 508: 170-174.

19 Surewicz W.K. \& Apostol M.I. 2011. Prion protein and its conformational conversion: a structural perspective. Topics in Current Chemistry. 305: 135-67.

20 Tranulis M.A, Benestad S.L., Baron T.\& Kretzschmar H. 2011. Atypical prion diseases in humans and animals. Topics in Current Chemistry. 305: 23-50.

21 Van Rheede T., Smolenaars M.M., Madsen O. \& De Jong W.W. 2003. Molecular evolution of the Mammalian prion protein. Molecular Biology and Evolution. 20: 111-121.

22 Vázquez-Fernández E., Alonso J., Pastrana M.A., Ramos A., Stitz L., Vidal E., Dynin I., Petsch B., Silva C.J. \& Requena J.R. 2012. Structural Organization of Mammalian Prions as Probed by Limited Proteolysis. PLoS One. 7(11): e50111. 
23 Vázquez-Fernández E., Vos M., Cebey L., Renault L., Sevillano A., Peters P.J., Fernández J.J., Young H., Wille H. \& Requena J.R. 2014. Recent advances towards an understanding of the structure of PrPSc. Prion. 8(1): 65-75.

24 Weissmann C., Enaria M., Klohn P.C., Rossia D. \& Flechsiga E. 2002. Transmission of Prions. The Journal of Infectious Diseases. 186: 157-165.

25 Weissmann C. 1996. Molecular biology of transmissible spongiform encephalopathies. FEBS Letter. 389: 3-11.

26 Westaway D., Zuliani V., Cooper C., Costa M.D., Neuman S., Jenny L., Detwiler L. \& Wilson R.T. 1984. The Camel. New York: MacGraw-Hill, pp.152-168.

27 Westergard L., Christensen H.M. \& Harris D.A. 2007. The cellular prion protein (PrPC): its physiological function and role in disease. Biochimica et Biophysica Acta. 1772(6): 629-644.

28 Wheeler J.C. 2012. South American camelids: past, present and future. Journal of Camelid Science. 5(1): 1-24

29 Wilson D.E. \& Reeder D.M. 2005. Mammal species of the world. Washington DC: Smithsonian Institution Press, pp.85-110.

30 Wopfner F., Weidenhofer G., Schneider R., von Brunn A., Gilch S., Schwarz T.F., Werner T. \& Schtzl H.M. 1999. Analysis of 27 mammalian and 9 avian PrPs reveals high conservation of flexible regions of the prion protein. Journal of Molecular Biology. 289: 1163-1168. 